\title{
Conocimientos y prácticas sobre la prevención de las infecciones respiratorias agu- das en las madres de niños menores de cinco años, atendidas en el Centro de Salud "Virgen del Rosario de Carapongo" Lima, julio de 2010
}

\author{
Nancy LUNA MAMANI' ${ }^{1}$ Edith LÓPEZ JURADO² y María Teresa CABANILLAS CHÁVEZ³
}

\begin{abstract}
RESUMEN
Objetivo: Evaluar los conocimientos y prácticas sobre la prevención de las infecciones respiratorias agudas en las madres de niños menores de 5 años atendidas en el Centro de Salud Virgen del Rosario de Carapongo, 2010. Metodología: El estudio es de tipo descriptiva, transversal. La población estudiada fueron 130 madres con hijos menores de 5 años. Se utilizó como técnica la entrevista, y como instrumento el cuestionario, aplicándoles en forma personal a cada madre por parte de las investigadoras. Resultados: Sobre el nivel de conocimiento, el 70\% de las madres de niños menores de cinco años tiene un nivel regular de conocimiento en cuanto a las infecciones respiratorias agudas; un $16.2 \%$ bueno y un $13.8 \%$ un nivel deficiente. En cuanto a las prácticas, el $52.1 \%$ de las madres de niños menores de cinco años tiene prácticas incorrectas en cuanto a la prevención de infecciones respiratorias agudas, mientras que un $47.8 \%$ si tiene prácticas correctas. Conclusiones: Se identificó que los conocimientos sobre la prevención de infecciones respiratorias agudas están relacionados con la edad y número de hijos. Asimismo, se determinó que no hay relación significativa entre conocimientos y prácticas. También se identificó que en las madres hay mayor disposición de aprendizaje sobre la prevención de infecciones respiratorias agudas, considerándose los niveles específicos de las variables predictora según la $(\mathrm{RM})$ razón de momios.
\end{abstract}

Palabras clave: Infecciones respiratorias agudas, conocimientos, prácticas y prevención.

\begin{abstract}
Objective: Assess knowledge and practices on the prevention of infections infections in mothers of children under 5 years treated at the Center Health Virgen del Carmen, 2011. Methods: The study is descriptive, transversal. The study populations were 130 mothers with children under 5 years. Technique was used as the interview, and as the survey instrument was used and applied in person to each mother by the researchers. Results: On the level of knowledge $70 \%$ of mothers of children under five has a regular level of knowledge regarding acute respiratory infections, $16.2 \% 13.8 \%$ good and a poor level. As for practices, $52.1 \%$ of mothers of children under five are improper practices in the prevention of acute respiratory infections while $47.8 \%$ if you practice. Conclusions: Was identified that the knowledge about the prevention of acute respiratory infections are associated with age and number of children. It was also determined that there is no significant relationship between knowledge and practice. We also identified that there is greater willingness of mothers of learning about the prevention of acute respiratory infections considering the specific levels of the predictor variables according to (RM).
\end{abstract}

Keywords: Acute respiratory infections, knowledge, practices and prevention.

\footnotetext{
${ }^{1}$ Licenciada en Enfermería, Universidad Peruana Unión

${ }^{2}$ Licenciada en Enfermería, Universidad Peruana Unión

${ }^{3}$ Coordinadora de la Maestría de Enfermería, Escuela de Posgrado de la Universidad Peruana Unión
} 


\section{INTRODUCCIÓN}

Las infecciones respiratorias agudas (IRA) representan la principal causa de morbilidad en el mundo y la causa más frecuente por la cual se acude o se usa un servicio de salud en todos los países; se ha estimado que las infecciones respiratorias agudas (IRA) representan entre el 30 y el $50 \%$ de las visitas de los niños a los establecimientos de salud y entre el 20 y el $40 \%$ de las hospitalizaciones pediátricas en la mayoría de los países en vías de desarrollo, Torres (2010).

Las enfermedades agudas del aparato respiratorio se presentan en su mayoría causadas por infecciones, y en la actualidad se prefiere referirse a ellas como infecciones respiratorias agudas (Suárez, 1999). Se estima que alrededor de 4 millones de niños menores de cinco años mueren cada año por alguna infección respiratoria aguda (IRA) en todo el mundo y cerca del $80 \%$ de estas defunciones se deben a la neumonía. Gran parte de las muertes ocurren en países pobres, donde las enfermedades infecciosas y parasitarias siguen siendo la causa número uno de las defunciones infantiles en el tercer mundo. (Romero, 1999).

Actualmente en el Perú, las infecciones respiratorias agudas constituyen la primera causa de morbilidad y la segunda de mortalidad en los menores de cinco años. Según la dirección general de las personas del Ministerio de Salud (Oficina Ejecutiva de Estadísticas), en el año 2001, la tasa de mortalidad infantil registrada alcanza a 14 por cada 1000 nacidos vivos y a 500 por cada 100,000 nacidos vivos. Existen 4 aspectos importantes en el análisis de las infecciones respiratorias agudas (IRA): prevención, control de factores de riesgo, morbimortalidad y atención médica. Por eso es necesario, que la madre conozca las medidas preventivas a tener en cuenta en sus niños considerando los conocimientos y prácticas de la misma, contribuyendo de esta manera a reducir la morbimortalidad como también la complicación de las infecciones respiratorias agudas, mejorando de esta manera la calidad de vida de este grupo vulnerable. (Torres, 2010). En tal sentido, el estudio tuvo como objetivo evaluar los conocimientos y prácticas sobre la prevención de las infecciones respiratorias agudas en las madres de niños menores de 5 años atendidas en el Centro de Salud Virgen del Carmen.

\section{MATERIAL Y MÉTODOS}

El estudio es de tipo descriptivo de corte transversal. La población en estudio es de 130 madres de niños menores de cinco años considerando el criterio de inclusión y exclusión de la población, y se llevó a cabo en los meses de febrero y marzo del 2011. Se utilizó un cuestionario, elaborado por las investigadoras y validado por juicio de expertos, el cuestionario estuvo constituido por 29 preguntas en relación a los conocimientos y prácticas sobre la prevención de las infecciones respiratorias agudas; cada pregunta tuvo un valoración de dos puntos. Tuvo tiempo de duración de 10 minutos. La participación de la población contó con plena aceptabilidad, asegurando el carácter privado y la confidencialidad a través de la entrega y recojo del cuestionario, previa orientación y obtener un alto índice de respuestas.

El posterior análisis y tabulación de los datos obtenidos se realizó por medio del paquete estadístico SPSS versión 15.0 para Windows, posteriormente se presentó los resultados en tablas y/o gráficos tras la realización de las pruebas estadísticas pertinentes al presente tipo y diseño de investigación. Se utilizó la t de Student para determinar la significativamente de las medias correspondientes a grupos dependientes.

\section{RESULTADOS}

\section{Figura 1}

Nivel de conocimientos de las madres de niños menores de cinco años sobre la prevención de las infecciones respiratorias agudas del Centro de Salud Virgen del Carmen - Carapongo, febrero - marzo, 2011

\section{Nivel de Conocimientos}

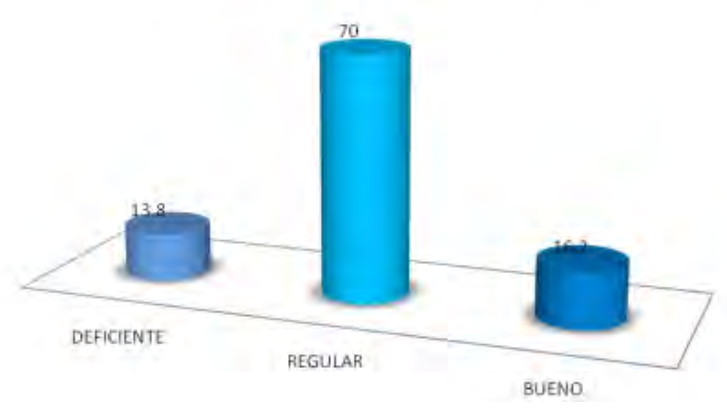


Como se observa en la figura 1; el 70\% de las madres de niños menores de cinco años tiene un nivel regular de conocimiento en cuanto a las infecciones respiratorias agudas, un $16.2 \%$ bueno y un $13.8 \%$ un nivel deficiente.

Figura 2 Prácticas de las madres de niños menores de 5 años sobre la prevención
de la IRA del Centro de Salud Virgen del Carmen - Carapongo febrero -
marzo 2011

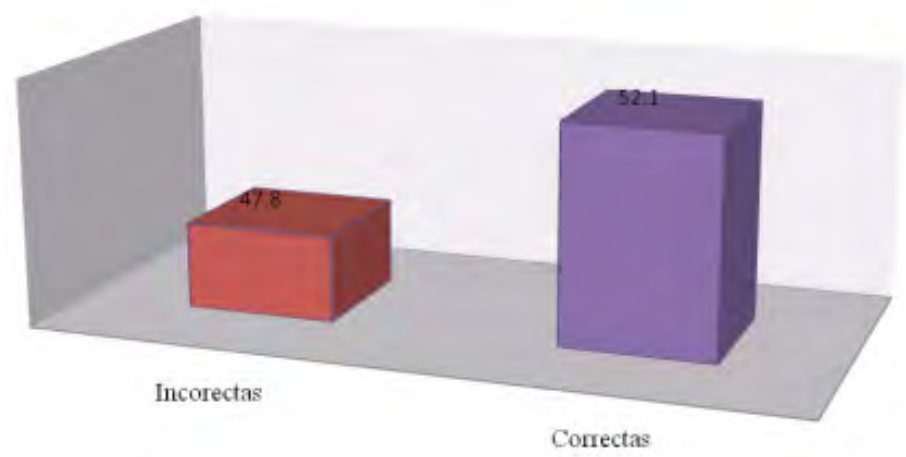

Se aprecia en la figura 2; que el 52.1\% de las madres de niños menores de cinco años tienen prácticas incorrectas en cuanto a la prevención de infecciones respiratorias agudas, mientras que un $47.8 \%$ tiene prácticas correctas.

\section{Figura 3}

Nivel de conocimientos y nivel de prácticas sobre las infecciones respiratorias agudas en las madres de niños menores de 5 años.

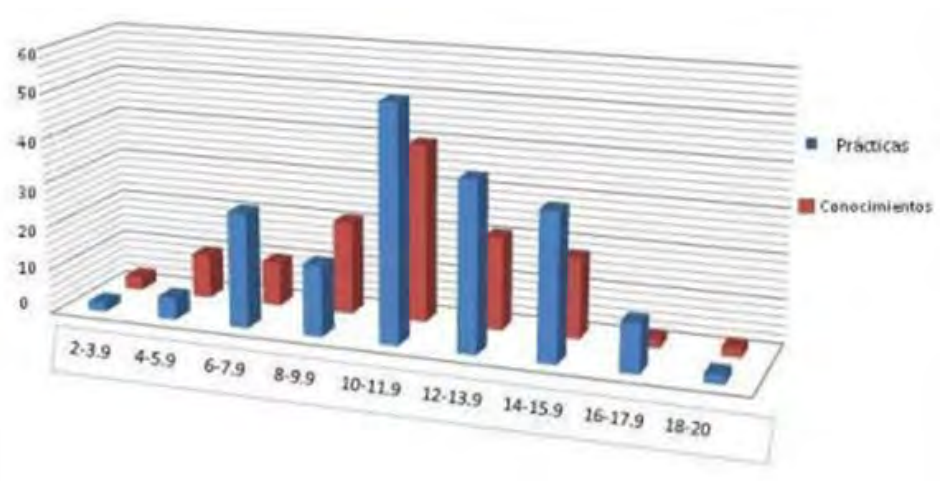

La media del nivel de conocimientos es de 11,6. Las notas de conocimiento tienen una mayor amplitud que la de prácticas $(\mathrm{DE}=3,1)$. La media del nivel de prácticas es de 10,7 y su desviación estándar es de 2,7. Se aprecia que los conocimientos y las prácticas tienen sus picos más altos en los rangos de 10 a 11,9.

\section{DISCUSIÓN}

Al evaluar los conocimientos y las prácticas sobre la prevención de infecciones respiratorias agudas se ubica en el nivel regular, seguido del nivel bueno y el nivel malo. Al respecto, Villapando (2010) sostiene que el conocimiento es un tipo de experiencia que contiene una representación de un hecho ya vivido, es la facultad consciente o proceso de comprensión, entendimiento que es propio del análisis, pensamiento, inteligencia y razón. Se le clasifica como conocimiento sensorial, respecto a la percepción de hechos externos y la capacitación de estados psíquicos internos. Conocimiento intelectivo, ello se origina de concepciones 
aisladas y de hechos casuales de ellas. Conocimiento de la razón, refiere a las causas internas fundamentales, generales, verdaderas de la existencia y modo de ser de las cosas.

En relación a los conocimientos que tiene la madre sobre los signos y síntomas de peligro, las madres relacionan a la fiebre como signo de cualquier infección; en este caso, de la infección respiratoria aguda. Según AEIPI (2011), los signos y síntomas de peligro en las infecciones respiratorias agudas son la: sibilancia, estridor, fiebre mayor de $38.5 \mathrm{C}^{\circ}$, taquipnea y tiraje intercostal.

En relación a los conocimientos de las madres acerca de los factores de riesgo por la que se presentan los problemas; $44.6 \%$ asocian al cambio de clima, el $15.4 \%$ menciona que el desabrigarse es un factor de riesgo, como también se observa que el $14.6 \%$ de las madres refieren que se debe a la deficiencia de alimentación y soló un $13.1 \%$ de las madres conoce los factores de riesgo como son la deficiencia de alimentación y la falta de vacunación. Estos elementos pueden ser adquiridos a través de la experiencia o de las creencias y tradiciones populares impuestas por la cultura; evidenciándose además, que las madres poseen poco conocimiento científico sobre los factores de riesgo de la IRA, las cuales deben ser impartidos por el personal de salud; en cuanto a las madres, si no tienen un claro conocimiento sobre los factores de riesgo de la IRA, entonces no pueden reconocer sus principales causas, por lo tanto, tampoco aplicarán las medidas adecuadas para la prevención de los problemas respiratorios.

Según Ticona (2009), los factores de riesgo son: deficiencia de alimentación, falta de vacunas, la contaminación ambiental y el humo de los cigarrillos, estos factores hacen que el niño pueda tener un problema.

Por otro lado, el nivel de práctica de las madres sobre la prevención de infecciones respiratorias agudas, muestra que la mayoría de las madres tiene prácticas correctas, seguida de las prácticas incorrectas. Por ejemplo la mayoría de las madres acude a vacunar a sus niños. Entre las principales medidas preventivas de la IRA, están las inmunizaciones que protegen al niño de complicaciones producidas por enfermedades inmunes prevenibles por vacunas, haciendo resistente el organismo del niño frente a la presencia de estas enfermedades. Asimismo, la alimentación balanceada de acuerdo a la edad del niño es importante para la prevención de esta enfermedad. Abregú (1999), refiere que la lactancia materna puede proteger contra la IRA mediante un cierto número de mecanismos, incluyendo sustancias antivirales y antibacterianas, las células inmunológicamente activas y los estimulantes del sistema inmune de los infantes. Los bebes alimentados a pecho presentan también un mejor estado nutricional en los primeros meses de vida, lo que puede contribuir a la reducción en la incidencia y gravedad de las enfermedades infecciosas.

\section{CONCLUSIONES}

- $\quad$ El 70\% de las madres de niños menores de cinco años tiene un nivel regular de conocimiento sobre la prevención de infecciones respiratorias agudas.

- Se aprecia que el $52.1 \%$ de las madres con niños menores de cinco años tienen prácticas incorrectas en cuanto a la prevención de infecciones respiratorias agudas, mientras que un $47.8 \%$ tiene prácticas correctas.

- Se identificó que los conocimientos sobre la prevención de infecciones respiratorias agudas están relacionados con la edad y número de hijos.

- Se determinó que no hay relación significativa entre conocimientos y prácticas.

- Se identificó que en las madres existe una mayor disposición de aprendizaje sobre la prevención de infecciones respiratorias agudas, considerándose los niveles específicos de las variables predictivas según la $(\mathrm{RM})$ razón de momios. 


\section{REFERENCIAS BIBLIOGRÁFICAS}

1. Alpizar L., Medina, E. (2000). Fisiopatología de la fiebre. Revista Cubana Medica Militar. 49 -54.

2. Ausbel, D. (1995). Psicología Educativa. México: Trillas. S.A.

3. Bunge, M. (1985). La investigación cientifica. Madrid: España: Ariel S.A.

4. Romero, C., Hernández, Vaca, M., Bernal, D. (1999). Creencias sobre infecciones respiratorias agudas en niños menores de cinco años: Estudio etnográfico en dos comunidades indígenas del estado de Oaxaca.

5. Gonzales, E. (2009). Infecciones Respiratorias. Obtenido de http://ideascompilativas.blogspot. com/2009/06/contenidos-conceptualesprocedimentales.html.

6. Grover, O. E. (2001). Vacuna del niño. Revista de enfermería de vacunación de la Universidad Unión, 3-10.
7. Miller, D. (1984). Investigaciones y estrategias para el estudio de las infecciones respiratorias agudas de la infancia. Bol. of Sanit Panam, 3.

8. Moreno, Z. \& Jhon, D. (2010). El porqué de las del dolor de garganta. Revista médica sobre las infecciones respiratorias agudas, 20 -30.

9. Quiroz, C. R. (2001). Conocimiento y su relación con la práctica de las madres de niños menores de cinco años sobre la prevención de las IRA. 3-5.

10. Russel, B. (1998). El conocimiento Humano. Lima Quinta edición, Tourus S.A 1998 España.

11. Villapando, J. (2000). Ciencia y conducta humana. Barcelona: Orbis S.A.

12. Venegas, M., Baquero, H. (2000). Enfoque del paciente con cianosis. División de ciencias Posgrado de Pediatría Universidad del Norte, 16 -23 . 